\title{
Nexus of Risk: The Co-occurring Problems of Gender-based Violence, HIV and Drug Use Among Women and Adolescent Girls
}

\author{
Claudia Stoicescu, Ariel Richer and Louisa Gilbert
}

\section{Introduction}

Worldwide, women and adolescent girls who use drugs (WUD) face vast inequalities in health status and health care. Among the most treacherous of these is the epidemic of gender-based violence (GBV), which disproportionately affects WUD and exacerbates their risk of HIV infection.

GBV encompasses multiple forms of violence, including childhood sexual abuse (CSA), intimate partner violence (IPV), non-partner assault and trafficking (United Nations General Assembly, 1993). Although population-level data quantifying the extent to which GBV affects women who use drugs remains scarce, studies conducted across a range of international settings suggest that WUD experience levels of IPV and other forms of GBV between 5 and 24 times higher than national surveillance estimates of IPV/GBV among general female populations (Gilbert et al., 2015; Stoicescu et al., 2018). WUD are also routinely victimised by law enforcement. A multicity study in Ukraine found that $66 \%$ of women who inject drugs (WID) had been sexually abused by the police (compared to $43 \%$ of men who inject drugs) (Kutsa et al., 2016).

GBV, substance use and HIV tend to cluster together among marginalised women and girls so frequently that epidemiologists have described their common co-occurrence as the SAVA (substance abuse, AIDS and violence) syndemic. Originally defined by Singer (1996), the SAVA syndemic refers to a set of closely inter-twined and mutual enhancing health problems that significantly affect the

\footnotetext{
The Impact of Global Drug Policy on Women: Shifting the Needle, 49-57 Copyright $(2021$ by Claudia Stoicescu, Ariel Richer and Louisa Gilbert These works are published under the Creative Commons Attribution (CC BY 4.0) licence. Anyone may reproduce, distribute, translate and create derivative works of these works (for both commercial and non-commercial purposes), subject to full attribution to the original publication and authors. The full terms of this licence may be seen at http://creativecommons.org/licences/by/4.0/legalcode doi:10.1108/978-1-83982-882-920200008
} 
overall health status of a population within the context of a perpetuating configuration of noxious social conditions.

The clustering of GBV, substance use and HIV in women and girls - facilitated by structural contexts such as poverty, societal gender roles and expectations, and ubiquitous stigma and discrimination - has resulted in a public health and human rights crisis. WUD who experience GBV face cumulative health problems, including greater sexual and injecting risk behaviours that facilitate the transmission of blood-borne viruses (BBV), increased AIDS-related mortality, and worse treatment outcomes in access to and retention in medication treatment for opioid use disorder and harm reduction services (Hatcher, Smout, Turan, Christofides, \& Stockl, 2015).

\section{Syndemic and Synergistic Pathways Linking GBV, Drug Use and HIV Among Women and Girls}

There are multiple biological, behavioural, and structural pathways linking GBV, drug use and BBV infection. The pathways linking these afflictions are bidirectional and often intersectional (i.e. synergistic).

\section{GBV and Drug Use}

Longitudinal research has established causal, bidirectional relationships between different types of GBV and drug use, which may vary depending on the type of GBV and substance used and whether both partners are under the influence (Devries et al., 2013). For example, drug use in intimate partnerships is associated with elevated odds of experiencing violent assault and IPV victimisation can likewise trigger the onset of or increase in substance use (El-Bassel, Gilbert, Wu, Go, \& Hill, 2005).

Drug use - particularly involving alcohol, crack cocaine and stimulants - can heighten feelings of irritability, jealousy and paranoia among perpetrators of GBV, as well as impair women's judgement when faced with a potentially risky situation, for instance by limiting the ability to negotiate condom use (Choenni, Hammink, \& van de Mheen, 2017). In situations where women's intimate partners provide them with drugs, power differentials are intensified as there may be an implicit expectation from partners to have sex in return (Gilbert, El-Bassel, Schilling, Wada, \& Bennet, 2000). A recent article found that dependencies on partners for drugs are further associated with experiencing drug overdose among women (El-Bassel et al., 2019).

Many WUD also engage in transactional sex to provide for their families or support a steady drug supply for themselves and/or their intimate partners, which may exacerbate their vulnerabilities to GBV and HIV (Shannon et al., 2015). WUD who trade sex are also at increased risk of experiencing physical and sexual abuse perpetrated by the police (Cottler, O'Leary, Nickel, Reingle, \& Isom, 2014). 


\section{The Catalytic Role of Trauma}

Trajectories of trauma from CSA to experiencing violence in adulthood increase the likelihood of developing substance use disorders and acquiring HIV. Women who experience trauma, such as childhood neglect, emotional, physical, or sexual abuse, are more likely to suffer from depression, anxiety and post-traumatic stress disorder (PTSD), which in turn places them at higher risk of using substances as a means to cope. CSA is a strong driver for the SAVA syndemic, as it is a risk factor for initiating injection drug use, engaging in risky sex, HIV infection and lack of engagement in HIV care and treatment, and is closely associated with PTSD (Engstrom, El-Bassel, \& Gilbert, 2012; Hadland et al., 2012; Schwartz et al., 2014).

Meanwhile, the trauma associated with experiencing IPV may lead to depression, since traumatic events can trigger fear, stress and isolation (Hyde, Mezulis, \& Abramson, 2008). Depression can perpetuate HIV transmission among women because it often co-occurs with and has mutually reinforcing and mediating relationships with both drug use and IPV (Devries et al., 2013). In high-income settings, research indicates that the prevalence of depression among people who use heroin is between $16 \%$ and 44\%, and is twice as high among women than men (Sordo et al., 2012).

\section{GBV and HIV}

Meta-analytical evidence indicates robust, mutually causal associations between different types of GBV and HIV among women, linked by direct and indirect pathways (Li et al., 2014). Specifically, sexual IPV can directly facilitate BBV transmission via vaginal lacerations and abrasions resulting from aggressive sexual intercourse, which heighten the risk of contracting HIV and other sexually transmitted infections (STIs) (World Health Organization (WHO, 2005). Physical IPV increases risk for HIV by $28-52 \%$ among different populations of women, including samples of WUD (Li et al., 2014). Psychological and physical assault operate similarly by shifting relationship power dynamics in favour of the perpetrator and establishing a context of fear and submission, which in turn increases the likelihood for engaging in condomless sex (Kouyoumdjian et al., 2013). Negotiating safer behaviours becomes more challenging where substance use is involved. The strongest effects on sexually transmitted HIV are those associated with amphetamine-type stimulants, particularly methamphetamine, which can facilitate sexual HIV transmission by heightening the sexual activity and increasing condomless sex while under the influence of drugs (Tavitian-Exley, Vickerman, Bastos, \& Boily, 2015).

The detrimental effects of IPV also extend to risky injecting practices. Psychological and physical IPV are significantly associated with higher odds of engaging in receptive syringe sharing (Stoicescu et al., 2018). The mechanism driving this association underscores the role of male power and control in many drug-involved relationships, especially in Southeast Asian contexts (Lazuardi et al., 2012), often sharing injecting equipment with an intimate partner tends to reinforce feelings of support, trust, love and intimacy (Simmons \& Singer, 2006). 


\section{Women at the Locus of Intersecting Risk}

The concentration of multiple disadvantages undermines the well-being of WUD, with women in low- and middle-income countries bearing the heaviest burden (Gilbert et al., 2015). Studies have documented increases in women's adverse health outcomes with the number of exposures to syndemic GBV, HIV and drug use and their mental health consequences. In a recent study conducted in Uganda, women reporting any two of depression, IPV, and/or alcohol use concurrently were six times more likely to test positive for HIV or an STI, compared with women without any exposures (Kiene, Lule, Sileo, Silmi, \& Wanyenze, 2017).

Empirical research has also shown that GBV, HIV, drug use and mental health problems interact synergistically to amplify health adversity beyond the independent effects of each affliction. In a study conducted in Indonesia, WWID who reported both depression and crystal meth were three times as likely to report symptoms associated with STIs. Those exposed to concurrent IPV and depression faced added harmful consequences, with the joint effect of these two risk factors resulting in a nearly threefold increase in women's prevalence of STI symptoms beyond each exposure's main effect (Stoicescu, Ameilia, Irwanto, Praptoraharjo, \& Mahanani, 2019).

\section{Risk Environments Fuelling the SAVA Syndemic}

Large-scale social forces operating at the community and structural levels - drug legislation, poverty, mass incarceration, beliefs about gender roles, and limited economic opportunities for women - play a crucial role in producing, perpetuating (and potentially ameliorating) syndemic risk factors in the context of GBV and HIV (Tsai, Mendenhall, Trostle, \& Kawachi, 2017). For example, male sex partners and/or drug dealers often control both access to and administration of drugs. In exchange for access to drugs, resources for shelter and safety, and to avoid arrest and prosecution in punitive legal environments, women may need to acquiesce to high-risk practices that may jeopardise their health and well-being. Such circumstances can create a dependency on both the substance itself and on the persons controlling access to it, thus indirectly shaping women's choices.

Moreover, the criminalisation of drug use and sex work can marginalise and drive women away from health and support services due to fear of arrest, and may also increase their exposure to police abuse. One study of WUD in community corrections in the United States found that $25 \%$ of women reported being coerced by police to trade sex in exchange for lessening charges and release from detention (Cottler et al., 2014). Fear of arrest and police abuse further impedes women from seeking help and protection from violent partners, further increasing their risk of GBV.

\section{What Works to Address the SAVA Syndemic Among Women?}

In the past decade, a continuum of multilevel integrated interventions and policies have shown success in targeting the specific behavioural, biological, and 
structural mechanisms that drive the SAVA syndemic. These include: (1) IPV screening, brief intervention and referral to treatment and services (SBIRT) models that may be integrated within a continuum of HIV/HCV test and treat interventions; (2) integrated behavioural IPV and HIV prevention interventions; (3) extended trauma-informed treatments to prevent HIV or promote medication adherence; and (4) community level and structural models.

\section{Integrating SBIRT Models Within a Continuum of HIV and HCV Test-and-Treat Interventions}

Controlled studies on behavioural IPV or GBV prevention interventions, which include IPV SBIRT, show that such models effectively reduce HIV risks and physical IPV for women in health care settings (Eckhardt et al., 2013). However, few existing models focus specifically on WUD, and even fewer address underserved and disproportionately affected subpopulations of women, such as adolescent girls, sexual minority women and racial/ethnic minority women.

The Women Initiating New Goals for Safety (WINGS) intervention, originally developed by the Social Intervention Group at the Columbia University School of Social Work, is an evidence-based intervention showing reductions in GBV and IPV among WUD around the world (Gilbert et al., 2017; GoddardEckrich et al., 2016). WINGS has been implemented as part of a wide range of services and programmes in six countries and in eight languages. Its recent implementation in three HIV-affected regions of India was integrated with existing harm reduction services, including the provision of HIV testing and treatment, overdose management, screening for tuberculosis, $\mathrm{HCV}$, sexual and reproductive health, and drug dependence treatment. Linking women with HIV and HCV testing and care was integral to the success of the project, which saw statistically significant reductions in women's exposure to physical and economic violence and engagement in unsafe injection practices after six months, as well as increases in $\mathrm{HIV} / \mathrm{HCV}$ testing and treatment uptake.

From Case to Cause: Scaling up a Brief Intervention 'WINGS' to Identify and Tackle GBV among Women Who Use Drugs in Kyrgyzstan

In 2013, a group of harm reduction advocates, providers, and researchers established a community collaborative research board (CCRB) to document and address the widespread problem of GBV against WUD in Kyrgyzstan. Led by GLORI, an NGO focussed on harm reduction service research, the CCRB received funding from the Open Society Foundations to adapt, implement, and evaluate a two-session IPV and GBV screening, brief intervention, and referral to treatment (SBIRT) model (WINGS) with HIV counselling and testing among a pilot sample of seven WUD in Kyrgyzstan using a pre/post-design. 
At baseline, $73 \%$ of the women reported experiencing any physical or sexual IPV victimisation in the past year, and $60 \%$ reported any physical or sexual victimisation by a non-intimate partner (GBV). Half of the participants reported ever experiencing police violence. At the three-month follow-up, participants reported experiencing 59\% fewer physical IPV incidents in the prior 90 days than at baseline $(P<0.001)$ and $27 \%$ fewer physical GBV incidents $(P<0.01)$ (Gilbert et al., 2017). From baseline to the three-month follow-up, participants also reported a $65 \%$ reduction in the odds of using any drugs $(P<0.05)$ and were more likely to report receiving GBV-related services $(P<0.001)$. Over the next five years, the CCRB expanded the implementation of WINGS to six NGOs in Bishkek and Osh, the two largest cities in Kyrgyzstan. The CCRB focused on providing intensive capacity building with the domestic violence crisis centres to expand their counselling and residential services to WUD and to develop a coordinated care response to WUD experiencing GBV with key community stakeholders, including WUD with lived experience of GBV, police, harm reduction providers, emergency care providers, and social services.

\section{Integrated IPV, HIV and Substance use Prevention Interventions}

Evidence from the United States and Sub-Saharan Africa suggests that reducing the risk of IPV also reduces the risk of acquiring HIV, HCV, and other STIs (Gilbert et al., 2016; Montgomery et al., 2018; Wechsberg et al., 2019). With SBIRT models, such interventions have the greatest impact when effectively integrated with HIV prevention and treatment, including harm reduction and drug treatment initiatives and naloxone distribution for the prevention of overdose.

A computerised, group-based HIV and IPV prevention intervention among 306 WUD mandated to community corrections in New York significantly lowered risks of different types of IPV (Gilbert et al., 2016). Compared with control participants, women who participated in WORTH were $62 \%$ less likely to report experiencing any physical IPV at the 12-month follow-up, $76 \%$ less likely to report injurious IPV, and 78\% less likely to report forced sex. Among Hispanic women in the United States, SEPA (Salud/Health, Educación/Education, Promoción/ Promotion, y/ and Autocuidado/Self-care), a five-session, culturally specific, group-based HIV-risk reduction intervention, has effectively reduced IPV, alcohol intoxication and depressive symptoms, and improved partner communication compared with controls, both at trial stage (McCabe, Gonzalez-Guarda, Peragallo, \& Mitrani, 2015) and when implemented in real world settings (Peragallo et al., 2018).

Women's CoOp (WC), an integrated intervention consisting of two sessions designed to reduce substance use and sexual risk, was originally developed in North Carolina, U.S. with African American women who use drugs. It has since 
been classified by the U.S. Centers for Disease Control and Prevention as a 'bestevidence' behavioural intervention for HIV prevention. A recent adaptation, the Women's Health CoOp Plus, was efficacious in reducing HIV risk, physical and sexual violence, and heavy alcohol use, as well as boosting linkages to HIV care among women who use drugs in Pretoria, South Africa (W. Wechsberg et al., 2019).

Couples-based HIV prevention interventions may be more effective than individual-based interventions for promoting risk reduction within intimate relationships; however, few such interventions focus specifically on WUD and their partners. The Couples' Health CoOp, a South African couple-based HIV prevention intervention that targets alcohol and other drug use to reduce sexual risk and GBV and adaptation of the WC, offers alternatives for conflict resolution and promote healthy relationships was recently pilot-tested with promising results (Wechsberg et al., 2015).

\section{Trauma-informed Interventions}

Trauma-informed interventions target syndemic mechanisms linking substance use, child sexual abuse, PTSD- and GBV-related trauma, and HIV risks. Evidence from meta-analyses indicates that addressing trauma symptoms optimises other syndemic health outcomes, including reducing drug-associated risks, violence victimisation and risky sexual behaviour (Hobbs, Kushner, Lee, Reardon, \& Maurer, 2011; Torchalla, Nosen, \& Rostam, 2012). Integrated approaches that address several syndemic exposures concurrently are more likely to be effective at reducing trauma symptoms, improving other health outcomes, and are more cost-effective and responsive to client needs (Najavits \& Hien, 2013). Traumainformed interventions involve multi-session treatment elements such as psychoeducation and cognitive behavioural strategies, including problem-solving and coping skills building.

Seeking Safety, the most widely tested trauma-focused integrated intervention, has significantly reduced substance use and PTSD symptoms in a range of populations, including incarcerated women and women in drug treatment (King, 2015; Sabri \& Gielen, 2017). INSPIRE (Integrating Safety Promotion with HIV Risk Reduction), a brief intervention for WUD who trade sex, has shown improvement in increasing safety behaviours, condom negotiation self-efficacy, use of sexual violence support services, and reduction of transactional sex under the influence (Decker et al., 2017). Another integrated trauma-informed sexual risk reduction intervention for women with a history of CSA in the US reduced condomless sex with a primary partner, alcohol use, and IPV (Senn, Braksmajer, Urban, Coury-Doniger, \& Carey, 2017).

A trauma-focused adaptation of the WC targeting incarcerated women with a history of IPV in the U.S. resulted in significant reductions in women's condomless sex, PTSD symptoms, and depressive symptoms, as well as improved access to community resources (Johnson et al., 2015). Other recent adaptations showing promising results include expanding the Women's Health Co-Operative, an evidence-based HIV prevention programme, to include trauma-related 
psychoeducation, cognitive-behavioural strategies for coping with trauma and substance use cravings, social support strategies, and self-care components among young South African women at risk of HIV (Myers, Carney, Browne, \& Wechsberg, 2018).

\section{Community-level and Structural Interventions}

Reversing the key mechanisms of the SAVA syndemic at the population level requires community interventions targeting primary prevention of GBV and HIV as well as long-term cultural, economic, and/or legislative interventions. Tackling social and structural conditions driving the SAVA syndemic could achieve more efficient reductions of the disease burden wrought by syndemics compared with behavioural interventions alone (Tsai \& Burns, 2015).

Community mobilisation interventions are increasingly recognised as critical to generating zero tolerance for GBV environments, norms and behaviours. Research conducted mostly in Africa and India suggests that over time such interventions can successfully achieve reductions in GBV, as well as HIV and STI incidence. A community intervention in South Africa successfully modified negative gender norms among men, showing potential to reduce community tolerance for GBV, which may also prevent HIV (Pettifor et al., 2018). The Tathmini GBV study in Tanzania evaluated a comprehensive health facility and community-based programme and found that it contributed to community-wide positive changes including less tolerance for certain forms of violence, more gender-equitable norms, improved GBV knowledge, increased community actions to address violence, and increased utilisation of GBV services (Settergren et al., 2018).

Less research focuses on interventions that mitigate the adverse effects of nonpartner violence. A community intervention with female sex workers (FSWs) in Karnataka, India has shown significant reductions in experiencing rape in the past year, being arrested, and being beaten in the past six months by a non-partner (Beattie et al., 2015). Given the high rates of police violence faced by WUD, this remains a neglected and urgent area for intervention focus.

Comprehensive HIV and GBV prevention strategies must include structural solutions such as enhanced economic opportunities for women, which can contribute to reductions in multiple syndemic mechanisms simultaneously, as has been shown by interventions in South Africa and the US (Pronyk et al., 2006; Sherman, German, Cheng, Marks, \& Bailey-Kloche, 2006). JEWEL, an economic empowerment and HIV prevention intervention for WUD and FSW comprised of six 2-hour sessions on HIV risk reduction and the production and sale of jewellery, was associated with reductions in substance use and the number of transactional partners (Sherman et al., 2006).

\section{Opportunities for Future Research, Interventions and Policies}

In the past two decades, accumulating research has articulated mechanisms driving the SAVA syndemic among women and girls and identified key areas of 
intervention. Yet, significant gaps persist. By and large, existing epidemiological and intervention research targeting the nexus of syndemic risk among WUD remains focused in the US and, to a lesser extent, Sub-Saharan Africa. However, since 2015, a growing number of studies have been conducted in previously under-represented regions, including South and Southeast Asia and Central Asia.

Several populations of marginalised women at high risk of syndemic comorbidities, including transgender women and adolescent girls, remain largely invisible in the literature. There is an urgent need for research and practice to better understand how the syndemic is playing out among these groups and how it intersects with the positionality of ethnic/racial minority women. All future research and implementation must meaningfully engage affected women and girls in the design, implementation, and evaluation of initiatives that concern them.

Structural interventions, including community mobilisation, economic empowerment, and drug policy reforms may have the greatest population-level impact on redressing syndemic harms, yet remain grossly overlooked and underresearched. An integrated, impactful response to these intersecting epidemics must include the decriminalisation of drug use and sex work as necessary conditions for reducing stigma, redressing the high levels of police violence against WUD, and broadening access to GBV, substance use and harm reduction services.

The disease burden wrought by syndemics among WUD can only be reduced through a comprehensive, integrated continuum of multi-level interventions that are supported by conducive policies. 